\title{
PHÁT TRIỂN CHƯƠNG TRÌNH ĐÀO TẠO THEO HƯỚNG TIẾP CẬN CDIO ĐỐI VỚI KHỐI KINH TẾ TẠI TRƯờnG ĐẠI HỌC THỦ DÂU MỘT
}

\author{
Nguyễn Ngọc Mai ${ }^{(*)}$ \\ (*) Tiến sĩ. Truờng Đại học Thủ Dầu Một. Email: mainn@tdmu.edu.vn
}

DOI: $10.37550 /$ tdmu.CFR/2021.01.108

\section{Tóm tắt}

Khoa Kinh tế Truò̀ng Đại học Thủ Dầu Một đã áp dụng chuong trình đào tạo theo đề xuoóng CDIO làm bối cảnh đào tạo sinh viên tù năm 2016. Khoa Kinh tế đã áp dụng CDIO trong việc phát triển chưong trình đào tạo cho cả 6 chưong trình; cu thể là Quản trị kinh doanh, Kế toán, Tài chính ngân hàng, Quản lý công nghiệp, Logistic và quản lý chuối cung úng, Du lịch. Trong quá trình xây dựng và phát triển chưong trình đào tạo, chúng tôi đã thực hiện khảo sát các bên liên quan để xác định chuẩn đầu ra của chương trình đào tạo, thiết kế chwơng trình giảng dạy hoà hợp tích cưc đem đến kết quả học tập mong đợi đáp úng chuẩn đầu ra. Bên cạnh đó, tất cả các chuơng trình đều áp dụng dạy hoc phần Nhập môn ngành cho sinh viên năm nhất, nhằm giới thiệu cho sinh viên một cái nhìn tổng thể về ngành nghề mà mình theo hoc, để sinh viên được trải nghiệm thưc tế ngay tù năm đầu tiên. Tù đó, giúp sinh viên có đam mê và nhiệt huyết đối với ngành nghề đã chọn. Sinh viên được trải nghiệm không gian khởi nghiệp và sáng tạo ngay tại truờng. Bài viết giới thiệu việc áp dụng 12 tiêu chuẩn CDIO vào việc cải tiến chuơng trình đào tạo của khối ngành kinh tế.

Từ khóa: kinh tế, 12 tiêu chuẩn CDIO, nhập môn ngành

\section{1. Đặt vấn đề}

Theo triết lý CDIO là đổi mới cách xây dựng chương trình đào tạo theo cách tiếp cận năng lực nhằm đảm bảo chất lượng đầu ra, đào tạo ra những sinh viên phát triển toàn diện, đáp ứng nhu cầu của xã hội Việt Nam nói riêng và hội nhập quốc tế nói chung. CDIO lần đầu tiên được áp dụng trong khối ngành kỹ thuật cơ khí. Do đó, việc cải tiến một số chương trình dựa trên hình thành ý tưởng, thiết kế ý tưởng, thực hiện và vận hành, đã được thực hiện rộng rãi trong lĩnh vực đào tạo kỹ thuật (Crawley và cộng sự, 2007). Tuy nhiên, hiện nay CDIO không chỉ giới hạn ứng dụng trong các chương trình kỹ thuật. Nó cũng được áp dụng cho các chương trình phi kỹ thuật. Doan et al. (2014) đề xuất các tiêu chuẩn CDIO cho các lĩnh vực khác. Ngoài ra, Malmqvist (2015) đã tiết lộ một kinh nghiệm thực tế về cách chuyển các tiêu chuẩn CDIO sang các bối cảnh các ngành phi kỹ thuật. Nghiên cứu này minh họa cách áp dụng CDIO trong lĩnh vực kinh tế nhằm cải thiện đầu ra cho sinh viên. Đầu tiên, tác giả mô tả 
việc triển khai và kinh nghiệm của các chương trình áp dụng CDIO. Phần tiếp theo sẽ trình bày các nghiên cứu điển hình trong các chương trình thuộc khoa kinh tế.

\section{Phương pháp tiếp cận theo CDIO}

Kết quả đầu ra của chương trình Tài chính ngân hàng là tạo ra những sinh viên tốt nghiệp chuyên nghiệp thực hành đáp ứng các yêu cầu công nghiệp và xã hội. Các tiêu chuẩn CDIO cho thấy các kết quả cao trong việc nâng cao chất lượng giáo dục. Như đã nêu trong bối cảnh $\mathrm{CDIO}$ (tiêu chuẩn $1 \mathrm{CDIO}$ ), đề xướng $\mathrm{CDIO}$ đã được trình bày và giới thiệu cho tất cả các giảng viên từ năm 2013 và Đại học Thủ Dầu Một (ĐHTDM) gia nhập tổ chức CDIO thế giới từ năm 2015. Từ đó, các chương trình đào tạo của khoa được phát triển dựa theo đề xướng CDIO. Sinh viên được giảng viên điều phối học tập theo hướng giải quyết vấn đề và hoàn thành kế hoạch học tập của mình theo các giai đoạn của CDIO. Bên cạnh đó, không ngừng nâng cao năng lực giảng dạy của giảng viên, tăng cường các giảng viên có kinh nghiệm nâng cao $\mathrm{CDIO}$ và sự hài lòng của các bên liên quan về không gian làm việc và học tập tại ĐHTDM. Cuộc khảo sát các bên liên quan được thực hiện để thu thập về sự đóng góp của từng môn học vào chuẩn đầu ra của chương trình đào tạo.

\begin{tabular}{|c|l|c|c|c|c|c|c|c|c|c|c|c|}
\hline STT & $\begin{array}{c}\text { Học phần phụ } \\
\text { trách }\end{array}$ & HK & ELO1 & ELO2 & ELO3 & ELO4 & ELO5 & ELO6 & ELO7 & ELO8 & ELO9 & ELO10 \\
\hline 1 & KT & 1.1 & $\mathrm{~S}$ & & $\mathrm{~S}$ & & & $\mathrm{H}$ & & $\mathrm{S}$ & & $\mathrm{S}$ \\
\hline 2 & MK CB & 1.1 & $\mathrm{~S}$ & & & $\mathrm{H}$ & $\mathrm{H}$ & $\mathrm{S}$ & & & $\mathrm{H}$ & \\
\hline 3 & NMTCNH & 1.1 & $\mathrm{H}$ & & & $\mathrm{S}$ & $\mathrm{S}$ & & & & $\mathrm{H}$ & $\mathrm{S}$ \\
\hline 4 & PLDC & 1.1 & $\mathrm{~S}$ & & & & $\mathrm{~S}$ & $\mathrm{~S}$ & & & $\mathrm{H}$ & $\mathrm{S}$ \\
\hline 5 & TCC1 & 1.1 & $\mathrm{~S}$ & & $\mathrm{~S}$ & & & & & $\mathrm{~S}$ & & $\mathrm{~S}$ \\
\hline 6 & NCKH & 1.2 & $\mathrm{~S}$ & & $\mathrm{~S}$ & & $\mathrm{H}$ & & & $\mathrm{S}$ & & $\mathrm{S}$ \\
\hline 7 & KTVM & 1.2 & $\mathrm{H}$ & $\mathrm{S}$ & & $\mathrm{S}$ & & $\mathrm{S}$ & & & & $\mathrm{S}$ \\
\hline 8 & LKT & 1.2 & $\mathrm{H}$ & & & & $\mathrm{S}$ & $\mathrm{S}$ & & & $\mathrm{H}$ & $\mathrm{S}$ \\
\hline 9 & NLTKKT & 1.2 & $\mathrm{H}$ & & & $\mathrm{S}$ & $\mathrm{S}$ & & & $\mathrm{S}$ & & $\mathrm{S}$ \\
\hline 10 & TCC2 & 1.2 & $\mathrm{~S}$ & & $\mathrm{~S}$ & & & & & $\mathrm{~S}$ & & $\mathrm{~S}$ \\
\hline 11 & NLKT & 1.3 & $\mathrm{~S}$ & & $\mathrm{~S}$ & & & & $\mathrm{~S}$ & & $\mathrm{~S}$ & $\mathrm{~S}$ \\
\hline 12 & THNLKT & 1.3 & $\mathrm{H}$ & & $\mathrm{S}$ & & & & $\mathrm{S}$ & & $\mathrm{H}$ & $\mathrm{S}$ \\
\hline 13 & KTVM & 2.1 & $\mathrm{H}$ & $\mathrm{S}$ & & $\mathrm{S}$ & & $\mathrm{S}$ & & & & $\mathrm{S}$ \\
\hline 14 & PPNCKHTKT & 2.1 & $\mathrm{~S}$ & $\mathrm{~S}$ & & $\mathrm{H}$ & & & & $\mathrm{H}$ & & $\mathrm{H}$ \\
\hline 15 & QTH & 2.1 & $\mathrm{~S}$ & $\mathrm{~S}$ & & $\mathrm{~S}$ & $\mathrm{~S}$ & & & & & $\mathrm{~S}$ \\
\hline 16 & TCTT & 2.1 & $\mathrm{~S}$ & $\mathrm{~S}$ & & & $\mathrm{H}$ & $\mathrm{S}$ & & & & $\mathrm{S}$ \\
\hline 17 & TTC & 2.1 & $\mathrm{~S}$ & $\mathrm{~S}$ & $\mathrm{~S}$ & & $\mathrm{~S}$ & & & & & $\mathrm{~S}$ \\
\hline 18 & TDBLUD & 2.1 & & & & $\mathrm{H}$ & $\mathrm{S}$ & $\mathrm{S}$ & & $\mathrm{S}$ & & $\mathrm{H}$ \\
\hline 19 & KTTC & 2.2 & & $\mathrm{~S}$ & $\mathrm{~S}$ & & & & $\mathrm{~S}$ & & $\mathrm{~S}$ & \\
\hline 20 & QTHCVP & 2.2 & $\mathrm{~S}$ & $\mathrm{~S}$ & & $\mathrm{~S}$ & $\mathrm{H}$ & $\mathrm{S}$ & & & & \\
\hline
\end{tabular}


Trường Đại học Thủ Dầu Một - Trường Đại học Duy Tân NXB Tài Chính 2021

\begin{tabular}{|c|c|c|c|c|c|c|c|c|c|c|c|c|}
\hline 21 & THMLN & 2.2 & $\mathrm{H}$ & & & & & $\mathrm{S}$ & & & & $\mathrm{S}$ \\
\hline 22 & NVNHTM1 & 2.2 & & $\mathrm{~S}$ & $\mathrm{H}$ & $\mathrm{S}$ & & $\mathrm{S}$ & & & $\mathrm{H}$ & \\
\hline 23 & KTCTML & 2.3 & $\mathrm{H}$ & & & & & $\mathrm{S}$ & & & & $\mathrm{H}$ \\
\hline 24 & TCQT & 2.3 & $\mathrm{H}$ & $\mathrm{H}$ & & $\mathrm{S}$ & $\mathrm{S}$ & & & & $\mathrm{S}$ & \\
\hline 25 & TTCS & 2.3 & & $\mathrm{H}$ & & $\mathrm{S}$ & & $\mathrm{H}$ & & $\mathrm{S}$ & $\mathrm{H}$ & \\
\hline 26 & TCDN & 2.3 & & $\mathrm{H}$ & $\mathrm{H}$ & $\mathrm{S}$ & & & & $\mathrm{H}$ & & $\mathrm{S}$ \\
\hline 27 & LBCTC & 3.1 & & $\mathrm{H}$ & $\mathrm{H}$ & & & & $\mathrm{S}$ & & $\mathrm{H}$ & $\mathrm{S}$ \\
\hline 28 & NVNHTM2 & 3.1 & & $\mathrm{H}$ & & & $\mathrm{S}$ & & $\mathrm{H}$ & $\mathrm{S}$ & $\mathrm{H}$ & \\
\hline 29 & KTXHDNB & 3.1 & $\mathrm{H}$ & & & & & $\mathrm{S}$ & & & & $\mathrm{S}$ \\
\hline 30 & $\mathrm{~T}$ & 3.1 & & $\mathrm{H}$ & $\mathrm{H}$ & & $\mathrm{S}$ & $\mathrm{H}$ & & & $\mathrm{H}$ & \\
\hline 31 & PTHDKD & 3.1 & & $\mathrm{H}$ & & $\mathrm{H}$ & & & $\mathrm{H}$ & $\mathrm{S}$ & $\mathrm{H}$ & \\
\hline 32 & PTTC & 3.1 & & $\mathrm{H}$ & & $\mathrm{H}$ & & & $\mathrm{H}$ & $\mathrm{S}$ & $\mathrm{H}$ & \\
\hline 33 & NHMP1 & 3.2 & & & $\mathrm{H}$ & & $\mathrm{S}$ & $S$ & $\mathrm{H}$ & & $\mathrm{H}$ & \\
\hline 34 & CNXHKH & 3.2 & $\mathrm{H}$ & & & & & $S$ & & & & $\mathrm{H}$ \\
\hline 35 & KTNH & 3.2 & & $\mathrm{H}$ & & $\mathrm{S}$ & $\mathrm{S}$ & & $\mathrm{H}$ & & $\mathrm{H}$ & \\
\hline 36 & TCDN & 3.2 & & $\mathrm{H}$ & $\mathrm{H}$ & $\mathrm{S}$ & & & & $\mathrm{H}$ & & $\mathrm{S}$ \\
\hline 37 & TTQT & 3.2 & & $\mathrm{H}$ & & $\mathrm{S}$ & & & $\mathrm{H}$ & & $\mathrm{S}$ & $\mathrm{S}$ \\
\hline 38 & TDTD & 3.2 & & $S$ & & $\mathrm{H}$ & & & $\mathrm{H}$ & $\mathrm{S}$ & $\mathrm{H}$ & \\
\hline 39 & MKNH & 3.3 & & $\mathrm{H}$ & & $\mathrm{H}$ & $\mathrm{S}$ & & & $\mathrm{H}$ & $\mathrm{S}$ & \\
\hline 40 & NHTW & 3.3 & & & $\mathrm{H}$ & $\mathrm{H}$ & $\mathrm{S}$ & $\mathrm{S}$ & & & $\mathrm{H}$ & \\
\hline 41 & TCC & 3.3 & & & $\mathrm{H}$ & $\mathrm{H}$ & $\mathrm{H}$ & $\mathrm{S}$ & & & $\mathrm{H}$ & \\
\hline 42 & BH & 3.3 & & & & & $\mathrm{~S}$ & $\mathrm{H}$ & $\mathrm{H}$ & $\mathrm{S}$ & $\mathrm{H}$ & \\
\hline 43 & TCCTDQG & 3.3 & & & & & $S$ & $\mathrm{H}$ & $\mathrm{H}$ & $S$ & $\mathrm{H}$ & \\
\hline 44 & TTCK & 4.1 & & $S$ & $\mathrm{~S}$ & & $\mathrm{H}$ & & $\mathrm{H}$ & & $\mathrm{H}$ & \\
\hline 45 & TTTC & 4.1 & & $\mathrm{~S}$ & $\mathrm{~S}$ & & $\mathrm{H}$ & & $\mathrm{H}$ & & $\mathrm{H}$ & \\
\hline 46 & QTNHTM & 4.1 & & $\mathrm{H}$ & $\mathrm{H}$ & $\mathrm{H}$ & $\mathrm{S}$ & & & & $\mathrm{H}$ & \\
\hline 47 & QTRRTC & 4.1 & & & $\mathrm{H}$ & $\mathrm{H}$ & & & $\mathrm{H}$ & $\mathrm{S}$ & & $\mathrm{S}$ \\
\hline 48 & DTTC & 4.1 & & & & $\mathrm{H}$ & $\mathrm{S}$ & $\mathrm{S}$ & $\mathrm{H}$ & & $\mathrm{H}$ & \\
\hline 49 & QTDMDT & 4.1 & & & & $\mathrm{H}$ & $\mathrm{S}$ & $\mathrm{S}$ & $\mathrm{H}$ & & $\mathrm{H}$ & \\
\hline 50 & TTHCM & 4.1 & $\mathrm{H}$ & & & & & $S$ & & & & $\mathrm{H}$ \\
\hline 51 & NHMP2 & 4.2 & & & & $\mathrm{H}$ & & $\mathrm{H}$ & $\mathrm{H}$ & $\mathrm{H}$ & $\mathrm{H}$ & \\
\hline 52 & LSD & 4.2 & $\mathrm{H}$ & & & & & $S$ & & & & $\mathrm{H}$ \\
\hline 53 & TTTN & 4.2 & & & $\mathrm{H}$ & & & $\mathrm{H}$ & $\mathrm{H}$ & $\mathrm{H}$ & $\mathrm{H}$ & \\
\hline 54 & BCTN & 4.3 & & & $\mathrm{H}$ & $\mathrm{H}$ & & & & $\mathrm{H}$ & $\mathrm{H}$ & $\mathrm{H}$ \\
\hline
\end{tabular}


Nhằm giới thiệu kiến thức và nền tảng cho sinh viên tài chính ngân hàng, đồng thời đáp ứng tiêu chuẩn $4 \mathrm{CDIO}$, môn học nhập môn ngành được giới thiệu ngay từ đầu năm 1 cho sinh viên, mời các chuyên gia trong lĩnh vực tài chính ngân hàng đến chia sẻ về yêu cầu kiến thức, kỹ năng, thái độ đối với sinh viên tốt nghiệp ngành tài chính ngân hàng và cơ hội nghề nghiệp của ngành tài chính ngân hàng. Bên cạnh đó, sinh viên được trải nghiệm sản phẩm thực tế của lĩnh vực tài chính ngân hàng bao gồm trải nghiệm sản phẩm tại ngân hàng, sản phẩm bảo hiểm và thực hiện giao dịch tại sàn giao dịch chứng khoán.

Các kinh nghiệm hình thành - thiết kế - thực hiện - vận hành được thể hiện trong các lớp học này. Trong giai đoạn hình thành, sinh viên học cách đáp ứng nhu cầu và mong đợi của người nhận hoặc khách hàng, các yếu tố môi trường ảnh hưởng và cách giải quyết vấn đề bằng giải pháp phù hợp. Lên kế hoạch, lên kịch bản đi thực tế... được đề xuất trong giai đạo thiết kế. Trong giai đoạn thực hiện, sinh viên chuyển đổi thiết kế của họ thành sản phẩm. Cuối cùng, các sản phẩm được giao cho giảng viên sau khi đi thực tế trong giai đoạn vận hành.

Trong tiêu chuẩn 5 của CDIO, để nâng cao kinh nghiệm về thiết kế và xây dựng, bên cạnh đó, sinh viên được trải nghiệm với học phần mô phỏng, học phần cho làm các project môn học. Các học phần này cho sinh viên học tập trên phần mềm mô phỏng và mời chuyên gia đến hướng dẫn sản phẩm cụ thể, sinh viên đến nơi làm việc của các đối tác để được hướng dẫn sản phẩm và sinh viên thực hiện project với sản phẩm được hướng dẫn đó 1 cách thực tế.

Chương trình đào tạo cung cấp cho sinh viên các môn học đáp ứng tiêu chuẩn 7 của CDIO, học tập trải nghiệm tích hợp, bên cạnh đó ĐHTDM cũng đã đào tạo giảng viên giảng dạy theo phương pháp hoà hợp tích cực (tiêu chuẩn $8 \mathrm{CDIO}$ ) nhằm đáp ứng việc giảng dạy hoà hợp tích cực cho sinh viên. Sinh viên có nhiều học phần thực hành, thực tập thực tế tại các đơn vị, từ đó họ có cơ hội tích hợp kiến thức, kỹ năng, thái độ vào thực tế. Đánh giá học tập sử dụng nhiều phương pháp phù hợp với kết quả học tập (tiêu chuẩn $11 \mathrm{CDIO}$ ). Các giảng viên đã và đang làm việc trong các chương trình đào tạo của khoa Kinh tế không ngừng nâng cao năng lực của giảng viên (tiêu chuẩn $9 \mathrm{CDIO}$ ). Để nâng cao năng lực giảng dạy (tiêu chuẩn CDIO 10), các giảng viên phải tham gia ít nhất một khóa đào tạo mối năm. ĐHTDM hàng năm đều có tổ chức nhiều khoá đào tạo cho giảng viên đăng ký học vào những thời gian phù hợp cho giảng viên và hoàn toàn miễn phí. Để theo dõi sự cải tiến liên tục của việc thực hiện $\mathrm{CDIO}$, các giảng viên trong khoa đã tiến hành tự đánh giá chương trình bằng cách sử dụng các phiếu đánh giá nhằm cải tiến theo đúng lộ trình của Trường đề ra, có phản hồi tích cực cho giảng viên và sinh viên (đáp ứng tiêu chuẩn $12 \mathrm{CDIO}$ ).

\section{3. Áp dụng học tập trải nghiệm vào môn học cụ thể - bảo hiểm}

Đây là môn học dành cho sinh viên năm 3 ngành Tài chính ngân hàng. Trong môn học này sinh viên có thể tiếp cận các sản phẩm bảo hiểm, quản lý tốt nhóm làm việc, tiếp cận các phương tiện để có thể học tập một cách tích cực và đa dạng.

Hình thành: Trong lớp học, nhiều phương pháp dạy và học được áp dụng, ví dụ như thảo luận, động não, học tập dựa trên vấn đề, đóng vai, làm việc nhóm. Tác giả nhận thấy rằng phương pháp mạnh mẽ nhất là một mô hình hướng dẫn về học tập tương tác và hợp 
tác. Phương pháp này được áp dụng với một dự án hợp tác trong một phần của một học và cuối môn học. Là một dự án hợp tác, một nhóm sinh viên được giao vai trò như một một tư vấn viên để mô tả về việc tư vấn khách hàng thông qua hoạt động trải nghiệm thực tế của nhóm. Sinh viên được đến công ty bảo hiểm đã ký kết hợp tác với ĐHTDM để được hướng dẫn cụ thể về các sản phẩm bảo hiểm.

Thiết kế: Sau khi phân tích yêu cầu của khách hàng mục tiêu, từng nhóm lên kế hoạch giới thiệu về sản phẩm dịch vụ sao cho có sức hấp dẫn khách hàng để khách hàng hiểu về ưu điểm của sản phẩm đó và thuyết phục khách hàng tham gia sản phẩm đó.

Thực hiện: Mỗi nhóm sinh viên thiết kế brochure sản phẩm để giới thiệu và thực hiện các bảng minh hoạ cho khách hàng xem

Vận hành: Quá trình làm việc nhóm đạt được hiệu quả cao, vì việc tiếp cận khách hàng và thuyết phục đối tượng khách hàng khác nhau tham gia sản phẩm của nhóm. Và nhóm có thể giải quyết các vấn đề phát sinh trong quá trình thực hiện project.

\section{Kết luận}

Bài viết này đã chỉ ra hướng tiếp cận CDIO cho khối ngành kinh tế, và cụ thể là ngành Tài chính ngân hàng thuộc khoa Kinh tế, nhằm đáp ứng nhu cầu xã hội cho sinh viên tốt nghiệp ngành Tài chính Ngân hàng. Thông qua đó, giới thiệu một môn học cụ thể học tập trải nghiệm thực tế tại doanh nghiệp, từ đó sinh viên có cái nhìn thực tế hơn về nghề nghiệp và học tập một cách tích cực hơn.

\section{Tài liệu tham khảo}

[1] Crawley, E., Malmqvist, J., Ostlund, S., and Brodeur, D. (2007). Rethinking engineering education. The CDIO Approach, 302, 60-62.

[2] Doan, T. T. M., Nguyen, N. H., Ngo, T. D., Tran, H. V., Nguyen, C. Q., Mai, T. T. (2014). The results and achievements of five years in applying CDIO: From pilot to widespread implementation. Proceedings of the 2014' CDIO Conference, Ho Chi Minh City.

[3] Malmqvist, J. (2015). Applying the CDIO approach to non-engineering education. CDIO Asian Regional Meeting, Ho Chi Minh City. 